\title{
Role of planktonic and sessile extracellular metabolic byproducts on Pseudomonas aeruginosa and Escherichia coli intra and interspecies relationships
}

\author{
Susana Patrícia Lopes • Idalina Machado • \\ Maria Olívia Pereira
}

Received: 25 March 2010/ Accepted: 26 July 2010/Published online: 3 September 2010

(C) Society for Industrial Microbiology 2010

\begin{abstract}
Bacterial species are found primarily as residents of complex surface-associated communities, known as biofilms. Although these structures prevail in nature, bacteria still exist in planktonic lifestyle and differ from those in morphology, physiology, and metabolism. This study aimed to investigate the influence of physiological states of Pseudomonas aeruginosa and Escherichia coli in cell-to-cell interactions. Filtered supernatants obtained under planktonic and biofilm cultures of each single species were supplemented with tryptic soy broth (TSB) and used as the growth media (conditioned media) to planktonic and sessile growth of both single- and two-species cultures. Planktonic bacterial growth was examined through $\mathrm{OD}_{640}$ measurement. One-day-old biofilms were evaluated in terms of biofilm biomass (CV), respiratory activity (XTT), and CFU number. Conditioned media obtained either in biofilm or in planktonic mode of life triggered a synergistic effect on planktonic growth, mainly for $E$. coli single cultures growing in $P$. aeruginosa supernatants. Biofilms grown in the presence of $P$. aeruginosa biofilms-derived metabolites presented less mass and activity. These events highlight that, when developed in biofilm, $P$. aeruginosa release signals or metabolites able to prejudice single and binary biofilm growth of others species
\end{abstract}

This article is part of the BioMicroWorld 2009 Special Issue.

S. P. Lopes - I. Machado - M. O. Pereira

IBB-Institute for Biotechnology and Bioengineering,

Centre for Biological Engineering, Universidade do Minho,

Campus de Gualtar, 4710-057 Braga, Portugal

M. O. Pereira ( $\square)$

Centro de Engenharia Biológica, Universidade do Minho,

Campus de Gualtar, 4710-057 Braga, Portugal

e-mail: mopereira@deb.uminho.pt and of their own species. However, products released by their planktonic counterparts did not impair biofilm growth or activity. E. coli, living as planktonic or sessile cultures, released signals and metabolites or removed un-beneficial compounds which promoted the growth and activity of all the species. Our findings revealed that inter and intraspecies behaviors depend on the involved bacteria and their adopted mode of life.

Keywords Interspecies signaling - Synergistic and antagonistic interactions · Planktonic growth · Biofilm growth

\section{Introduction}

In nature, bacteria live by interacting and communicating with each other, regardless if they belong to the same (intraspecies) or different species (interspecies). One of the major mechanisms of cell-cell communication in bacteria involves the synthesis and release of chemical molecules called diffusible signal molecules [50]. These signals can be cell-density related (quorum sensing-QS) or produced by bacteria at different stages of growth. Primary and secondary metabolites are recognized to contribute to a wealth of interactions between organisms [10] and can include a variety of nutrients, toxic or neutral metabolic byproducts, antibiotics, and other signaling molecules. Such products are released and accumulated in the surrounding environment during bacterial growth [13] and can induce expression of certain genes and/or physiological changes in neighboring cells $[14,32]$. The properties of these signals and the response elicited by them are important in ensuring bacterial survival and propagation in natural environments where hundreds of bacterial species 
coexist [17]. Responses of bacteria to chemical signals are quite varied and can include synergistic and/or antagonistic effects. Most research into interspecies bacterial interactions has focused on the beneficial aspects of these relationships that may include coaggregation $[18,26,31,38$, 43] and conjugation [15]. These positive interactions give advantages to microorganisms through the transfer of chemical signals, exchange of genetic information [33], growth promotion and increase of metabolic activity [42], and protection from adverse environmental conditions [23]. Positive interactions among competitors can even contribute to biodiversity [16]. However, not all interactions are beneficial, since antagonistic interactions play an important role in bacterial species predominance. Competition for substrate is considered to be the major evolutionary driving force in the microbial world [44]. Negative interactions can give rise to sporulation, suppression of respiration [12], growth inhibition through the production, for instance, of antimicrobial compounds, as antibiotics [35, 47]. For instance, lactic acid bacteria are known to produce some substances with antimicrobial activity, including antimicrobial peptides, which are able to inhibit foodborne pathogens and spoilage bacteria [7].

Most studies have reported cell-to-cell communication mediated by small diffusible molecules produced only by bacteria living in planktonic conditions. However, bacteria living in a biofilm differ from their planktonic counterparts, cohabiting as single cells, not only at the morphological level but also in their physiological and metabolic state [19]. These differences in the cell's physiological state are reflected by substantial changes in their gene expression pattern $[3,39,40]$, and may even induce quite varied responses in other bacteria.

In order to investigate whether bacteria lifestyle influences the type of signals and metabolites released into the surrounding environment, the effects of those products secreted by two important human-associated pathogen and commensal, $P$. aeruginosa and $E$. coli, living as free-floating cells and in biofilms, were analyzed. The role of such molecules was evaluated in cell suspensions and in biofilms of single and dual-species cultures formed by both strains.

\section{Materials and methods}

Bacterial strains and culture conditions

Pseudomonas aeruginosa ATCC 10145 (American Type Culture Collection) and Escherichia coli K12 MG1655 were used throughout this work. The strains were streaked and grown overnight at $37^{\circ} \mathrm{C}$ from a frozen stock on a nutrient agar plate (Tryptic soy agar, TSA, Merck, Portugal). Several colonies from each strain were used to inoculate a culture in $30 \mathrm{ml}$ of tryptic soy broth (TSB, Merck, Portugal) that was incubated at $37^{\circ} \mathrm{C}$, under agitation (130 rpm) for $12-18 \mathrm{~h}$.

Preparation and collection of supernatants of $P$. aeruginosa and E. coli

Supernatants were obtained from planktonic (planktonic supernatants) and sessile cultures (biofilm supernatants) of each bacterium. In order to collect the planktonic supernatants, overnight cultures of $P$. aeruginosa and $E$. coli in TSB were centrifuged $\left(7,000 \times g ; 4^{\circ} \mathrm{C} ; 5 \mathrm{~min}\right)$ and the crude supernatant was separated from the cells, filtered $\left(0.22-\mu \mathrm{m}\right.$ filter) and stored at $-20^{\circ} \mathrm{C}$ for further use. For collecting the biofilm supernatants, cells reserved above were used to form single biofilms. Briefly, cells were ressuspended in fresh TSB and the $\mathrm{OD}_{640}$ was measured until reaching a final concentration of $\sim 10^{7}$ cells $\mathrm{ml}^{-1}$. Both cultures were transferred to 96-well tissue culture plates (polystyrene, Orange Scientific, USA) $(200 \mu \mathrm{l}$ per well) and incubated at $37^{\circ} \mathrm{C}$ and $130 \mathrm{rpm}$, allowing biofilm formation for $24 \mathrm{~h}$. After this time, supernatants were recovered, filtered $(0.22-\mu \mathrm{m}$ filter $)$ and stored at $-20^{\circ} \mathrm{C}$. In order to test possible contamination, $10 \mu \mathrm{l}$ of filtered supernatants (from planktonic and biofilm cultures) from each species were plated on TSB agar plates.

Bacterial growth in the presence of supernatants

\section{Bacterial planktonic growth}

Previous cultures of $P$. aeruginosa and E. coli were centrifuged $\left(7,000 \times g ; 4^{\circ} \mathrm{C} ; 5 \mathrm{~min}\right)$ and ressuspended in TSB, until reaching $\sim 10^{7}$ cells $\mathrm{ml}^{-1}$ (by $\mathrm{OD}_{640}$ measurement). For dual-species cultures, a combination of $50 \%$ of suspended inocula of each species was used. These cell suspensions were diluted 1:2 in each supernatant, collected previously, transferred to microtiter plates $\left(200 \mu \mathrm{l}\right.$ culture per well), and incubated at $37^{\circ} \mathrm{C}$ with agitation of $130 \mathrm{rpm}$. Bacterial planktonic growth was followed by $\mathrm{OD}_{640}$ measurement at 1-h intervals, until reaching $24 \mathrm{~h}$. In order to validate the influence of supernatants in planktonic growth, normal growth curves in the presence of only TSB medium were also examined and used as a control test. All the experiments were repeated three times.

\section{Biofilm development and analysis}

Biofilms were developed according to the modified microtiter plate test proposed by Stepanović et al. [45]. The bacterial suspensions prepared above, were diluted 1:2 in each supernatant and transferred, under aseptic conditions, 
for a microtiter plate $(200 \mu \mathrm{l}$ per well). To promote biofilm formation, the plates were incubated aerobically on a horizontal shaker at $120 \mathrm{rpm}$, at $37^{\circ} \mathrm{C}$. After $24 \mathrm{~h}$, the content of each well was removed and the wells were washed twice with $250 \mu \mathrm{l}$ of sterile water. Biofilms were also formed in the presence of TSB (control), in order to compare with those grown in supernatants. The plates were air dried for $20 \mathrm{~min}$, and the remaining attached bacteria were analyzed in terms of biomass adhered on the surfaces of the microtiter plates and in terms of metabolic activity. The number of cultivable cells, by plate count agar, was also determined.

Biomass Biomass of single and dual-species biofilms were quantified by crystal violet $(\mathrm{CV})$ staining method adapted from Stepanović et al. [45]. Briefly, the 24-h biofilms formed within the 96 wells were fixed with $250 \mu$ of $98 \%$ methanol (Vaz Pereira, Portugal) per well for $15 \mathrm{~min}$. Afterwards, the plates were emptied and left to dry for $20 \mathrm{~min}$. Then, the fixed bacteria were stained for $5 \mathrm{~min}$ with $200 \mu \mathrm{l}$ of crystal violet (CV) (Merck, Portugal) per well. Excess stain was rinsed off by placing the plate under running tap water. After the plates were air dried, the dye bound to the adherent cells was resuspended with $200 \mu \mathrm{l}$ of $33 \%$ (v/v) glacial acetic acid (Merck, Portugal) per well. The optical density (OD) of the obtained solution was measured at $570 \mathrm{~nm}$ using a microtiter plate reader (Tecan, Model Sunrise-basic Tecan, Austria) and biofilm mass was presented as $\mathrm{OD}_{570}$ values. Control experiments to avoid false results were also performed in order to determine whether the tested media and the material of construction of the plates (polyester) could interact with $\mathrm{CV}$.

Respiratory activity The respiratory activity of single and mixed biofilms was measured through the XTT colorimetric method, as described by Stevens and Olsen [46], with some modifications. Biofilms were washed as described before and $200 \mu \mathrm{l}$ of a combined solution of XTT (Sigma-Aldrich) and PMS (phenazine methosulfate) (Sigma) was added to each well in order to obtain a final concentration of $150 \mu \mathrm{g} \mathrm{ml}^{-1}$ of XTT and $10 \mu \mathrm{g} \mathrm{ml}^{-1}$ of PMS. Afterwards, the plates were left to incubate for $3 \mathrm{~h}$, at $150 \mathrm{rpm}$, in the dark. The biofilm activity was determined through measurement of the content of each well by optical density at $490 \mathrm{~nm}\left(\mathrm{OD}_{490}\right)$ using a microtiter plate reader and the biofilm activity was presented as $\mathrm{OD}_{490}$. Control tests, using culture medium, the supernatants supplemented in TSB and empty wells, were also carried out, in order to avoid misleading results.

Cell culturability In order to determine the number of CFUs, biofilms formed in the presence of TSB and in the supernatants were removed by sonication and were subsequently serially diluted. After plating the serial dilution on TSA, the plates were incubated at $37^{\circ} \mathrm{C}$ in an aerobic incubator for $18 \mathrm{~h}$ prior to enumeration. The number of cultivable bacterial cells was determined separately and expressed as CFU per $\mathrm{cm}^{2}$.

Statistical analysis The data were analyzed using the Prism software package (GraphPad Software). One-way ANOVA tests were performed and $p<0.01$ was considered significant.

\section{Results}

Influence of the supernatants (planktonic vs. biofilm) of $P$. aeruginosa and $E$. coli on bacterial planktonic growth

After the supernatants from planktonic and biofilm cultures of $P$. aeruginosa and $E$. coli strains have been collected, they were used as growth media on planktonic growth of single and mixed cultures formed by both species. The planktonic development of all cultures was analyzed every hour, by $\mathrm{OD}_{640}$ measurement. Figure 1 presents the planktonic growth curves from single cultures of $P$. aeruginosa and $E$. coli and of their mixed cultures grown in the presence of planktonic and biofilm supernatants from each strain. Cultures grown in TSB medium were used as controls and are represented by the darkest lines. As can be observed, no supernatant showed ability to affect the growth of both cultures involving $P$. aeruginosa strain, either in single (Fig. 1a) or in mixed cultures (Fig. 1c), since the respective growth curves followed a pattern similar to the growth curve control. These results suggest that extracellular metabolites released either from $P$. aeruginosa and $E$. coli in distinct mode of life, were unable to affect the growth profile of single and mixed cultures with $P$. aeruginosa. This showed that the relationship established between both strains, in mixed cultures, was not disturb by any supernatant (planktonic or biofilm) from each strain. Conversely, single $E$. coli planktonic growth was greatly stimulated when grown in the $P$. aeruginosa biofilm supernatant (Fig. 1b), showing a synergistic response face to signaling molecules released from $P$. aeruginosa biofilms.

Influence of biofilm supernatants of $P$. aeruginosa and $E$. coli on single and mixed biofilms growth and activity

The effect of the supernatants obtained under biofilm conditions from $P$. aeruginosa and $E$. coli strains on their 
Fig. 1 Effect of the planktonic and biofilm supernatants on growth of planktonic single cultures of $P$. aeruginosa (a), E. coli $\mathrm{k}-12$ (b), and in mixed cultures of those bacteria (c). Values are means of three separate assays, and the bars indicate SD (a)

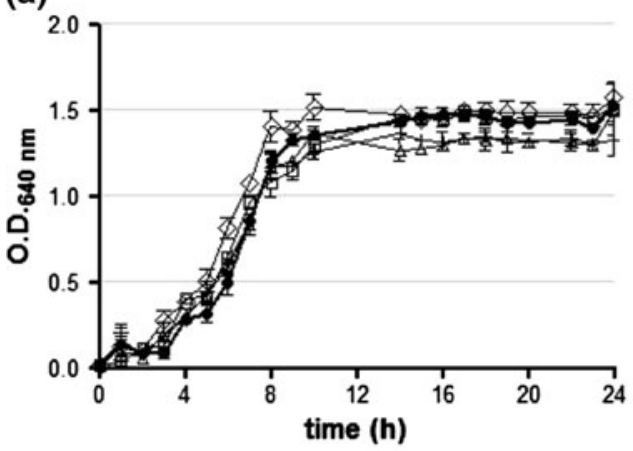

(b)

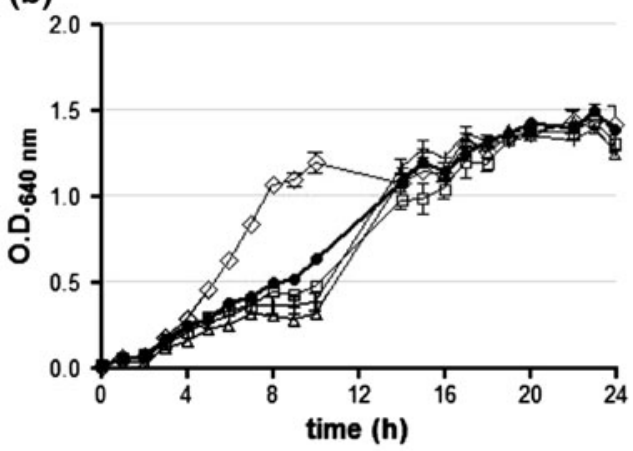

(c)

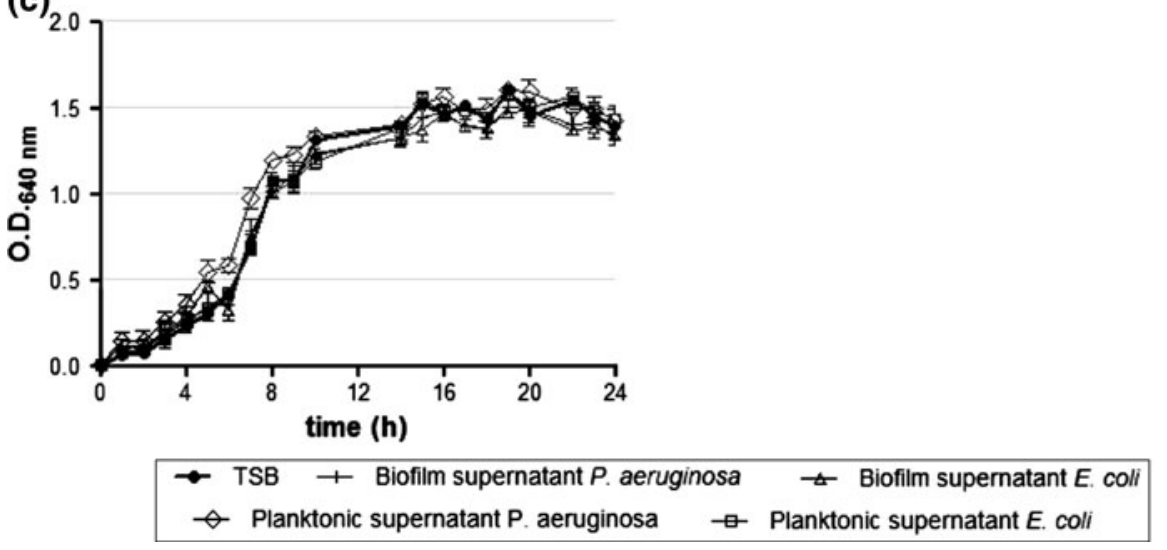

Fig. 2 Effect of biofilm supernatants from $P$. aeruginosa and $E$. coli on biofilm biomass (a) and respiratory activity (b) of their single and mixed biofilms. The values are means of three separate assays, and the bars indicate SD. $* p<0.01$ (vs. TSB) in one-way ANOVA test

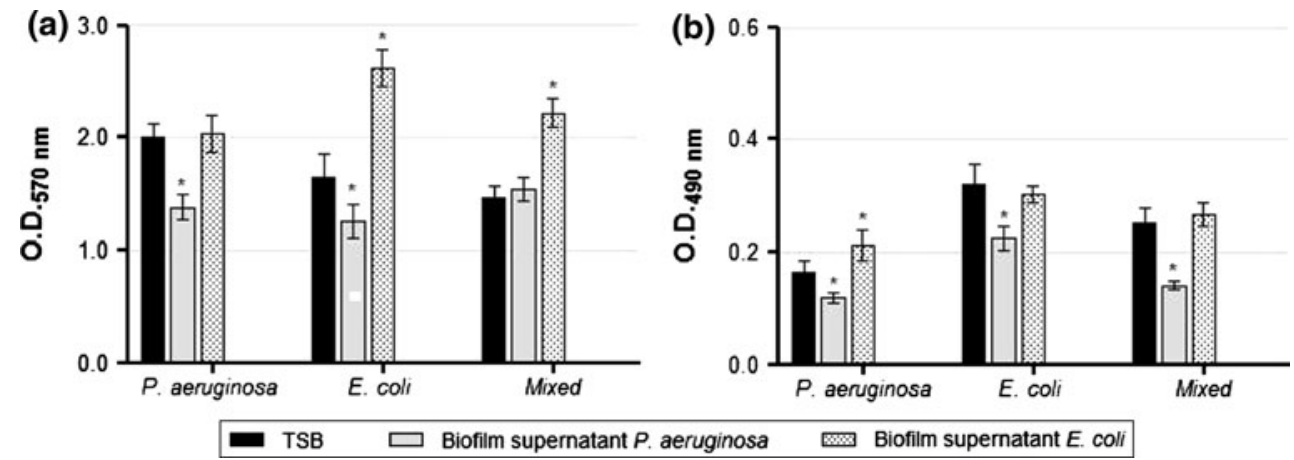

single and binary biofilms growth and activity are presented in Fig. 2. Although P. aeruginosa and E. coli are both Gram-negative bacteria, they trigger different effects both in biofilms growth and activity. As shown in Fig. 2a, biofilm supernatant of $P$. aeruginosa inhibited its own growth, as well as $E$. coli single biofilm $(p<0.01)$. However, no effect was observed on the formation of mixed biofilms formed by both strains grown in the presence of this supernatant in relation to the medium control. As can be seen by Fig. 2b, conditioned media produced during $P$. aeruginosa growth also showed the ability to disturb cell viability, including for dual-species biofilms, resulting in an antagonistic response by all sessile cultures. Concerning the biofilm supernatant of E. coli, signaling molecules produced by this species resulted in biofilms with equal or even more mass than those grown in TSB, mainly for itself (reaching an increase of about $60 \%$ on biomass) (Fig. 2a). It was also noticed a marked increase in dual-species growth $(p<0.01)$ caused by this supernatant, suggesting that modifications of the medium by $E$. coli living in biofilm, either by production of metabolites, signals, or removal of un-beneficial compounds, benefits its relationship with $P$. aeruginosa, promoting the development of mixed biofilm. However, when compared to control, those signals revealed not cause significant deviations in biofilms metabolic activity. Thus, in a general point of view, the biofilm supernatant of $E$. coli demonstrated a positive impact in all biofilms, mainly on biomass accumulation rather than respiratory activity. 
Influence of planktonic supernatants of $P$. aeruginosa and $E$. coli on single and mixed biofilms growth and activity

In Fig. 3 it is possible to observe the effect caused by planktonic supernant of $P$. aeruginosa and $E$. coli in monoand dual-species biofilm growth and respiratory activity. Unlike biofilm supernatants, these results demonstrated that metabolites released by each bacterium under planktonic conditions did not show antagonistic effect regarding the growth and activity of biofilms. Planktonic supernatant from $P$. aeruginosa showed ability to maintain the biomass accumulation in single biofilms, instead of reducing it, as observed in the presence of its biofilm supernatant. However, in the case of mixed biofilms, signal molecules produced by $P$. aeruginosa in planktonic lifestyle caused the stimulation on their growth $(p<0.01)$. Concerning the role of those products delivered from $P$. aeruginosa in planktonic state, on biofilms activity, no particular effect was detected, resulting in biofilms with similar activity as those grown in TSB. Nevertheless, biofilms grown in planktonic supernatant of $E$. coli had a synergistic response, resulting in biofilms with more biomass $(p<0.01)$. Similar results were detected on respiratory activity of all biofilms grown in that supernatant, resulting in more active biofilms, mainly for those formed by itself. The growth of mixed biofilm involving $P$. aeruginosa and $E$. coli was also promoted by extracellular metabolites from planktonic cultures of $E$. coli, suggesting that interactions between the species forming mixed biofilm were reinforced either by metabolites, signals released from E. coli in planktonic lifestyle or removal of un-beneficial compounds, resulting in more robust and more active biofilms.

Influence of supernatants (planktonic vs. biofilm) of $P$. aeruginosa and $E$. coli on cultivable cells

In order to complement biofilms growth and activity experiments, the number of cultivable cells within biofilms grown in the presence of supernatants from different lifestyle was also evaluated. In Fig. 4 it is possible to observe the cell numbers, in log, in single and mixed biofilms grown in supernatants from sessile and planktonic cultures of each species. The results obtained from these experiment showed a slight reduction in CFU number for biofilms grown in the presence of all supernatants, when compared to the control. However, reductions in cell number capable

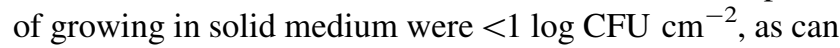
be observed in Fig. 4. The decrease was more evident for biofilms growing in the presence of supernatants resulting from sessile cultures, being more noticeable for $E$. coli biofilms-derived metabolites $(p<0.01)$. In fact, the number of cells within $P$. aeruginosa single biofilms decreased from $7.05 \log \mathrm{CFU} \mathrm{cm} \mathrm{cm}^{-2}$ (growing in TSB) to $6.44 \mathrm{log}$
Fig. 3 Effect of planktonic supernatants from $P$. aeruginosa and $E$. coli on biofilm biomass (a) and respiratory activity (b) of their single and mixed biofilms. The values are means of three separate assays, and the bars indicate $\mathrm{SD} . * p<0.01$ (vs. TSB) in one-way ANOVA test

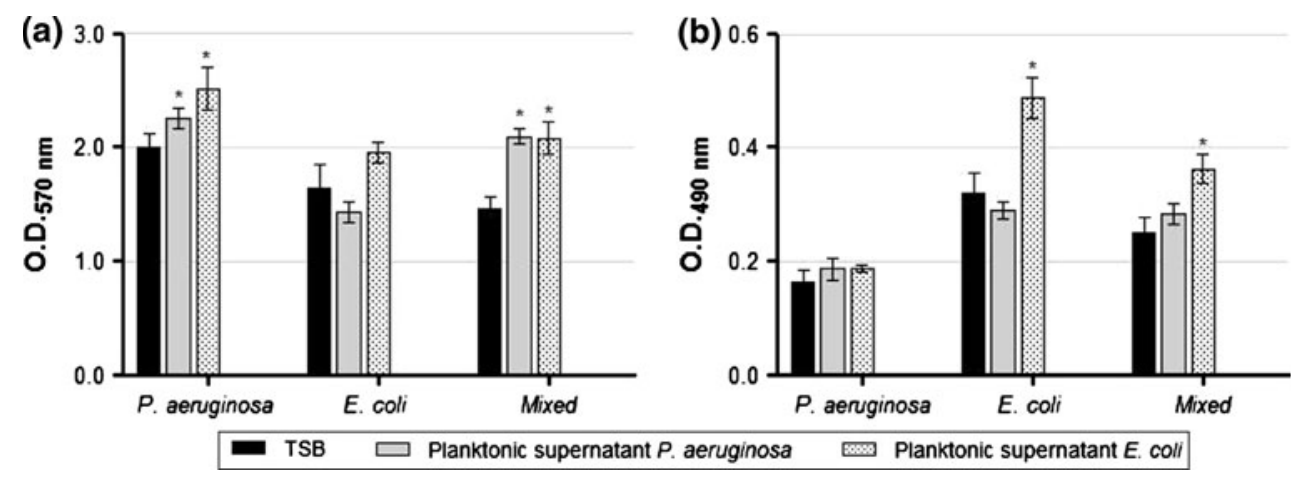

Fig. 4 Effect of biofilm (a) and planktonic supernatants

(b) from $P$. aeruginosa and E. coli on CFU number within their single and mixed biofilms. The values are means of three separate assays, and the bars indicate SD. $* p<0.01$ (vs.

TSB) in one-way ANOVA test (a)

(a)

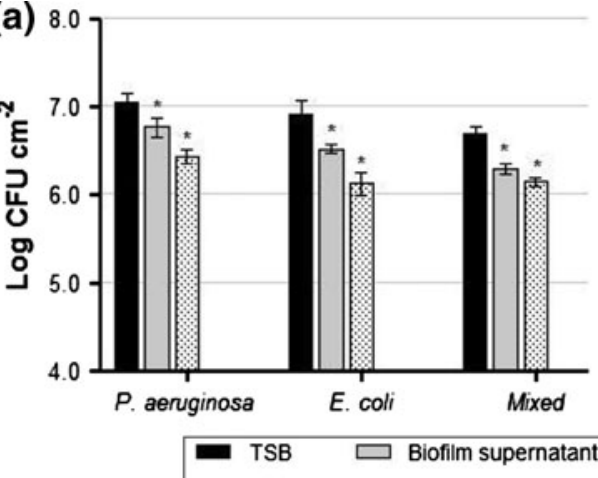

(b)
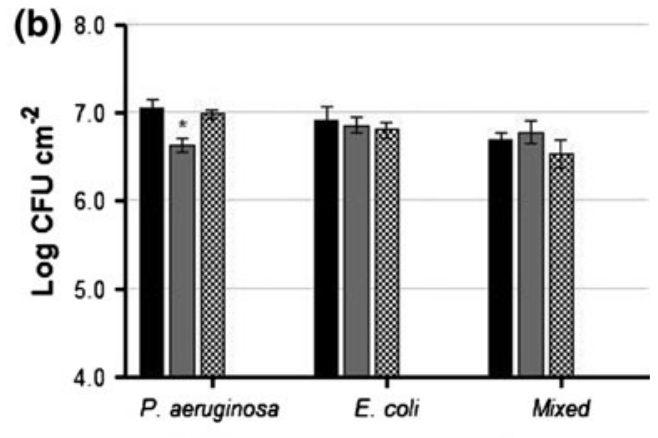

Biofilm supernatant $E$. coli Planktonic supernatant $P$. aeruginosa $\cong$ Planktonic supernatant $E$. coli 
CFU $\mathrm{cm}^{-2}$ (growing in TSB supplemented with E. coli biofilm supernatant). Cells from single $E$. coli biofilm presented a reduction from 6.92 to $6.12 \log \mathrm{CFU} \mathrm{cm} \mathrm{cm}^{-2}$, when co-cultured in its own biofilm supernatant. With $P$. aeruginosa as a partner, the $E$. coli numbers still showed a small decrease, reducing from 6.69 to 6.14 log $\mathrm{CFU} \mathrm{cm} \mathrm{cm}^{-2}$. Since biofilms growing in the presence of signaling molecules obtained from $E$. coli still remaining active, but with a reduced CFU number, this could indicate a loss of capability of cells to grow on agar.

\section{Discussion}

Interactions between microorganisms are well-known phenomena that allow a colony or a group of organisms to behave in a co-ordinated fashion to regulate processes contributing to virulence, antibiotic production, biofilm formation, and other developmental programs. Even though bacteria are more prevalent in nature living as communities, these structures are morphologically and physiologically differentiated from free-living bacteria, which may be reflected in the use of different signaling pathways. However, studies reporting cell-to-cell communication have only been directed to the effect of molecules produced by individuals in biofilms and the role of signaling molecules by planktonic bacteria still less well understood. The main objective of the current work was to compare the performance of signals or of metabolic by-products, produced in different physiological status of two major human-associated pathogens and commensal, $P$. aeruginosa and $E$. coli, in respect to their single and mixed planktonic growth and in biofilms.

Data obtained from planktonic growth experiments showed that the growth of $P$. aeruginosa single cultures and in combination with $E$. coli was not affected by the metabolites or signals molecules released by both strains. Moreover, both cultures presented a similar growth profile, suggesting that $E$. coli did not interfere with dual-species growth. Besides that, the use of $P$. aeruginosa planktonic supernatant as growth medium triggered acceleration on growth of $E$. coli, suggesting that metabolites secreted by $P$. aeruginosa in planktonic mode of life gave rise to a synergistic effect on $E$. coli monospecies culture. Based on these results, it is possible to state the ability of $P$. aeruginosa to promote the growth of other planktonic species. According to Shank and Kolter [42], many microorganisms can grow better in combination with others or in the presence of their partner's diffusible compounds. Conversely, Qin et al. [34] demonstrated that $P$. aeruginosa supernatant dramatically reduced Staphylococcus epidermidis growth in planktonic cultures. According to some authors [8, 22], P. aeruginosa possesses significant antibiotic activity against Gram-positive bacteria. The present study reinforces that statement since $E$. coli growth was favored by $P$. aeruginosa, revealing a synergistic response between both Gram-negative bacteria.

For this study, it was also proposed to evaluate the role of the signaling molecules released by bacteria in planktonic and sessile growth against single and mixed biofilms. Regarding the effect of biofilm supernatants on biofilm growth and activity, our findings revealed that extracellular molecules derived from single $P$. aeruginosa and $E$. coli populations showed distinct results on such parameters. In fact, signaling molecules confer to bacterial population the ability to instigate a collective behavioral change to environmental challenges [2]. According to some authors [9], $P$. aeruginosa is an opportunistic pathogen, which regulates an arsenal of extracellular virulence factors, using a complex hierarchical QS cascade involving AHL molecules. QS-regulated phenotypes can include, for example, the synthesis of antimicrobial compounds, such as antibiotics or rhamnolipid surfactants, which are believed to be involved in detachment of cells from the biofilm [4, 41] and may even interfere with biofilm formation of the producing species or others on its proximity. Thus, our data showed that the production of QS molecules do not always provided direct benefit to the producing bacteria, as hypothesized by some authors [11, 36, 37]. In E. coli, AHLs from other bacteria are sensed through the LuxR homologue (SdiA). This species does not synthesize such signals [27, 48], but those are recognized to reduce its biofilm formation [1, 20, 28]. However, the signals and metabolites released from $P$. aeruginosa biofilms did not disturb the growth of mixed biofilms. This may indicate that $P$. aeruginosa and $E$. coli form a mutual cooperation, protecting each other from hostile environmental conditions and therefore being more difficult its disruption. This synergistic interaction between both bacteria can be attributed to a strategy used by $E$. coli to interfere with $P$. aeruginosa signal molecules or metabolites. It is widely accepted that disruption or interference of AHL QS diminishes AHL-dependent expression of virulence determinants in producing bacteria [49]. Another explanation focuses on a kind of pact that could have been done between $E$. coli and $P$. aeruginosa, where the first provides nutrients and other substances to the second bacterium, while this strain does not disrupt the relationship between them.

Conversely to the effect provided by signal molecules or metabolites from $P$. aeruginosa, extracellular metabolites released by $E$. coli biofilms showed benefit in $P$. aeruginosa and $E$. coli single and mixed biofilms growth. In environmental and clinical settings, this becomes a serious problem, since these structures are prevalent and less susceptible to environmental stresses, giving rise to chronic 
infections that are notoriously difficult to eradicate [24, 25].

Although historically interspecies interactions have focused on growth interactions, the truth is that a variety of other phenotypic outcomes are possible to occur. Thus, the metabolic activity of biofilms developed in supernatants was also evaluated. Our findings revealed that the signals and metabolites produced by $P$. aeruginosa consortia or the removal of un-beneficial compounds triggered an inhibition in all biofilms activity, either in single or in binary mode of growth. As mentioned above, $P$. aeruginosa produces a myriad of metabolites, many of which with an observable bioactivity, such as antibacterial or phytotoxic activity [21], which may have been associated with disturbance on biomass and metabolic activity in single biofilms.

Concerning the role of planktonic supernatants in biofilms, results showed that unlike the effect produced by biofilm supernatants, mainly those from $P$. aeruginosa, any antagonistic responses were elicited by planktonic supernatants in biofilms biomass and activity. It is well recognized that bacteria in planktonic forms have unnatural and free access to nutrients, multiply rapidly and often are highly motile [30]. Growing freely in culture medium, there is no needs to bacteria establish antagonistic interactions and affect their and other species growth. Conversely, sessile bacteria growing on surfaces have nutrient limitations and so may grow more slowly and have restricted mobility [6]. To increase their chances of survival, bacteria compete with each other and start to release toxins and other antimicrobial compounds, which are able to interfere with their neighbors. These different signaling pathways used by bacteria in different mode of growth could be in the basis of distinct responses trigger in other bacteria. In this study, it was observed that metabolites secreted by $E$. coli, either in planktonic or in sessile life, always trigger positive interactions in $P$. aeruginosa, resulting in biofilms with more mass and activity.

In order to complement the previous results, we also evaluated the CFU number within biofilms growing in

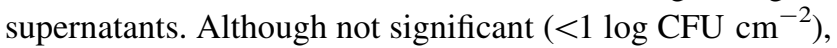
we found a decrease in cell number within biofilms growing in all supernatants, being more evident using biofilm supernatants as growth media, particularly from E. coli. Since biofilms growing in this supernatant presented metabolic activity, but a decline in cell number, it may be a sign of loss of the culturability feature of the bacterial cells. Many factors can be pointed out to justify the nonculturability of bacteria, namely lethal/sub-lethal injury of cells, adaptation and differentiation among others [5, 29]. This could even be related with a survival strategy used for bacteria that are introduced into a new environment.
Roszak and Colwell [37] coined the term "viable but nonculturable" for characterize those bacteria.

\section{Conclusions}

Bacteria are affected by the environment in which they live and the variety of other microbial species present. Interactions between microorganisms are well-known phenomena and represent a powerful selective force which has led to the evolution of a variety of effective strategies for colonizing and growing on surfaces. This work has focused on the influence of bacterial physiological state in cell-to-cell interactions. Bacteria living in distinct lifestyle lead to various and different behaviors on interspecies relationships, which suggests that the signals and metabolites produced in biofilm status are dissimilar from those delivered by their planktonic counterparts. Since most studies have been focused on interspecies communication mediated by individuals in biofilms, this study represents a novel area of interest, because although biofilm lifestyle takes prevalence in nature, bacteria in free suspension still exist and may even establish relationships between them.

Acknowledgments The authors acknowledge the financial support provided by the Portuguese Foundation for Science and Technology (grants: SFRH/BD/47613/2008 - Susana Lopes and SFRH/BD/ 20582/2004-Idalina Machado; and project PTDC/SAU-ESA/64609/ 2006).

\section{References}

1. Ahmer BM (2004) Cell-to-cell signalling in Escherichia coli and Salmonella enterica. Mol Microbiol 52:933-945

2. Atkinson S, Williams P (2009) Quorum-sensing and social networking in the microbial world. J R Soc Interface 6:959-978

3. Beloin C, Valle J, Latour-Lambert P, Faure P, Kzreminski M, Balestrino D, Haagensen JA, Molin S, Prensier G, Arbeille B, Ghigo JM (2004) Global impact of mature biofilm lifestyle on Escherichia coli K-12 gene expression. Mol Microbiol 51:659-674

4. Boles BR, Thoendel M, Singh PK (2005) Rhamnolipids mediate detachment of Pseudomonas aeruginosa from biofilms. Mol Microbiol 57:1210-1223

5. Colwell RR (2000) Bacterial death revisited. In: Colwell RR, Grimes DJ (eds) Nonculturable microorganisms in the environment. ASM, Washington, DC, pp 325-342

6. Costerton JW, Lewandowski Z, Caldwell DE, Korber DR, Lappin-Scott HM (1995) Microbial biofilms. Annu Rev Microbiol 47:711-745

7. de Carvalho KG, Bambirra FH, Kruger MF, Barbosa MS, Oliveira JS, Santos AM, Nicoli JR, Bemquerer MP, de Miranda A, Salvucci EJ, Sesma FJ, Franco BD (2009) Antimicrobial compounds produced by Lactobacillus sakei subsp. Sakei 2a, a bacteriocinogenic strain isolated from a Brazilian meat product. $\mathrm{J}$ Ind Microbiol Biotechnol (in press)

8. Deziel E, Lepine F, Milot S, He J, Mindrinos MN, Tompkins RG, Rahme LG (2004) Analysis of Pseudomonas aeruginosa 
4-hydroxy-2-alkylquinolines (HAQs) reveals a role for 4-hydroxy-2 in cell-to-cell communication. Proc Natl Acad Sci USA 101:1339-1344

9. Diggle SP, Gardner A, West SA, Griffin AS (2007) Evolutionary theory of bacterial quorum sensing: when is a signal not a signal? Phil Trans R Soc B 362:1241-1249

10. Duan K, Silbey CD, Davidson CJ, Surette MG (2009) Chemical interactions between organisms in microbial communities. Contrib Microbiol 16:1-17

11. Hense BA, Kuttler C, Müller J, Rothballer M, Hartmann A, Kreft JU (2007) Does efficiency sensing unify diffusion and quorum sensing? Nat Microbiol 5:230-239

12. Hoffman LR, Déziel E, D“Argenio DA, Lépine F, Emerson J, McNamara S, Gibson RL, Ramsey BW, Miller SI (2006) Selection for Staphylococcus aureus small-colony variants due to growth in the presence of Pseudomonas aeruginosa, vol 103, no. 52. PNAS (www.pnas.org)

13. Fuqua C, Greenberg EP (2002) Listening in on bacteria acylhomoserine lactone signalling. Nat Rev Mol Cell Biol 3:685-695

14. Fuqua WC, Winans SC, Greenberg EP (1996) Census and consensus in bacterial ecosystems: the LuxR-LuxI family of quorum sensing transcriptional regulators. Ann Rev Microbiol 50:727-751

15. Ghigo JM (2001) Natural conjugative plasmids induce bacterial biofilm development. Nature 412:442-445

16. Gross K (2008) Positive interactions among competitors can produce species-rich communities. Ecol Lett 11:929-936

17. Jayaraman A, Wood TK (2008) Bacterial quorum sensing: signals, circuits, and implications for biofilms and disease. Annu Rev Biomed Eng 10:145-167

18. Kolenbrander PE (2000) Oral microbial communities: biofilms, interactions, and genetic systems. Annu Rev Microbiol 54:413-437

19. Landini $P$ (2009) Cross-talk mechanisms in biofilm formation and responses to environmental and physiological stress in Escherichia coli. Res Microbiol 160(4):259-266

20. Lee J, Jayaraman A, Wood TK (2007) Indole is an interspecies biofilm signal mediated by SdiA. BMC Microbiol 7:42

21. Leisinger T, Margraff R (1979) Secondary metabolites of the fluorescent pseudomonads. Microbiol Rev 43:422-442

22. Lepine F, Milot S, Deziel E, He J, Rahme LG (2004) Electrospray/mass spectrometric identification and analysis of 4-hydroxy-2-alkylquinolines (HAQs) produced by Pseudomonas aeruginosa. J Am Soc Mass Spectrom 15:862-869

23. Leriche V, Briandet R, Carpentier B (2003) Ecology of mixed biofilms subjected daily to a chlorinated alkaline solution: spatial distribution of bacterial species suggests a protective effect of one species to another. Environ Microbiol 5:64-71

24. Lewis K (2007) Persister cells, dormancy and infectious disease. Nat Rev Micro 5:48-56

25. Mah T-F, Pitts B, Pellock B, Walker GC, Stewart PS et al (2003) A genetic basis for Pseudomonas aeruginosa biofilm antibiotic resistance. Nature 426:306-310

26. Malik A, Sakamoto M, Hanazaki S, Osawa M, Suzuki T, Tochigi M, Kakii K (2003) Coaggregation among nonflocculating bacteria isolated from activated sludge. Appl Environ Microbiol 69:6056-6063

27. Michael B, Smith JN, Swift S, Heffron F, Ahmer BM (2001) SdiA of Salmonella enterica is a LuxR homolog that detects mixed microbial communities. J Bacteriol 183:5733-5742

28. Moons P, Van Houdt R, Aertsen A, Vanoirbeek K, Engelborghs Y, Michiels CW (2006) Role of quorum sensing and antimicrobial component production by Serratia plymuthica in formation of biofilms, including mixed biofilms with Escherichia coli. Appl Environ Microbiol 72:7294-7300
29. Oliver JD (1995) The viable but non-culturable state in the human pathogen Vibrio vulificus. FEMS Microbiol Lett 133(3):203-208

30. Olson ME, Ceri H, Morck DW, Buret AG, Read RR (2002) Biofilm bacteria: formation and comparative susceptibility to antibiotics. Can J Vet Res 66(2):86-92

31. Palmer RJ Jr, Gordon SM, Cisar JO, Kolenbrander PE (2003) Coaggregation-mediated interactions of streptococci and actinomyces detected in initial human dental plaque. J Bacteriol 185:3400-3409

32. Parsek MR, Greenberg EP (2005) Sociomicrobiology: the connections between quorum sensing and biofilms. Trends Microbiol 13:27-33

33. Pesci EC, Milbank JB, Pearson EC, McKnight S, Kende AS, Greenberg EP, Iglewski BH (1999) Quinolone signaling in the cell-to-cell communication system of Pseudomonas aeruginosa. Proc Natl Acad Sci USA 96(20):11229-11234

34. Qin Z, Yang L, Qu D, Molin S, Tolker-Nielsn T (2009) Pseudomonas aeruginosa extracellular products inhibit staphylococcal growth, and disrupt established biofilms produced by Staphylococcus epidermidis. Microbiology 155:2148-2156

35. Rao D, Webb JS, Kjelleberg S (2005) Competitive interactions in mixed-species biofilms containing the marine bacterium Pseudoalteromonas tunicata. Appl Environ Microbiol 71:1729-1736

36. Redfield RJ (2002) Is quorum sensing a side effect of diffusion sensing? Trends Microbiol 10:365-370

37. Roszak DB, Colwell RR (1987) Survival strategies of bacteria in the natural environment. Microbiol Rev 51:365-379

38. Rickard AH, Gilbert P, High NJ, Kolenbrander PE, Handley P, McBain AJ, Ledder RG, Handley PS, Gilbert PS (2003) Coaggregation between freshwater bacteria within biofilm and planktonic communities. FEMS Microbiol Lett 220:133-140

39. Sauer K, Camper AK (2001) Characterization of phenotypic changes in Pseudomonas putida in response to surface-associated growth. J Bacteriol 183:6579-6589

40. Schembri MA, Kjaergaard K, Klemm P (2003) Global gene expression in Escherichia coli biofilms. Mol Microbiol 48:253-267

41. Schooling SR, Charaf UK, Allison DG, Gilbert P (2004) A role for rhamnolipid in biofilm dispersion. Biofilms 1:91-99

42. Shank EA, Kolter R (2009) New developments in microbial interspecies signaling. Curr Opin Microbiol 12:205-214

43. Sharma A, Inagaki S, Sigurdson W, uramitsu HK (2005) Synergy between Tannerella forsythia and Fusobacterium nucleatum in biofilm formation. Oral Microbiol Immunol 20:39-42

44. Simões LC, Simões M, Vieira MJ (2007) Microbial interactions in drinking water biofilms. In: Biofilms: coming of age. University of Manchester, Manchester, pp 43-52

45. Stepanović S, Vuković D, Davić I, Savić B, Ŝvabić-Vlahović M (2000) A modified microtiter-plate test for quantification of staphylococcal biofilm formation. J Microbiol Meth 40:175-179

46. Stevens MG, Olsen SC (1993) Comparative-analysis of using Mtt and $\mathrm{Xtt}$ in colorimetric assays for quantitating bovine neutrophil bactericidal activity. J Immun Meth 157(1-2):225-231

47. Tait K, Sutherland LW (2002) Antagonistic interactions amongst bacteriocin-producing enteric bacteria in dual-species biofilms. J Appl Microbiol 93:345-352

48. Van Houdt R, Aertsen A, Moons P, Vanoirbeek K, Michiels CW (2006) $N$-acyl-L-homoserine lactone signal interception by Escherichia coli. FEMS Microbiol Lett 256:83-89

49. Venturi V, Subramoni S (2009) Future research trends in the major chemical language of bacteria. HFSP J 3(2):105-116

50. Waters CM, Bassler BL (2005) Quorum sensing: cell-to-cell communication in bacteria. Annu Rev Cell Dev Biol 21:319-346 